\section{Clinical detection of schizophrenia-prone}

\section{individuals}

\author{
Critical appraisal
}

JOSEF PARNAS

\begin{abstract}
Summary Issues that undermine the early detection of schizophrenia include an inadequate grasp of the construct validity of the concept of schizophrenia, insufficient conceptualisation of psychosis and of the illness onset, and a general lack of a theoretical framework for

psychopathology. Subjective experience is emphasised as a potentially promising domain for future research.
\end{abstract}

\section{Declaration of interest None.}

This article focuses on the limitations of contemporary psychopathology which appear to diminish the feasibility of early detection and prevention of the schizophrenia-spectrum disorders. Basically, there are three options for early intervention: (a) intervening early in the course of psychosis, to reduce its duration and potential sequelae; (b) intervening in the 'prodromal' phase, to prevent or defer the onset of psychosis and/or attenuate its course; and (c) primary prevention, through reducing the prevalence of potential risk factors (e.g. obstetric complications). This paper discusses the first two issues only and abstains from epidemiological considerations on the flexibility of detection programmes and from a critical appraisal of the rationale behind such programmes.

Several psychopathological issues merit attention. First, schizophrenia remains a 'fuzzy' scientific concept. For example, the so-called polydiagnostic studies (e.g. comparing DSM-III (American Psychiatric Association, 1980), research diagnostic criteria with other, equally reasonable definitions of schizophrenia) consistently demonstrate that the number of patients with schizophrenia diagnosed in a given

†Paper presented at the Third International Early Psychosis Conference, Copenhagen, Denmark, September 2002 sample varies by a factor of $2-3$, depending on the diagnostic criteria applied and the composition of the examined sample (Jansson et al, 2002). The official endorsing of the DSM-III concept of schizophrenia and its contemporary permutations (DSM-IV (American Psychiatric Association, 1994) and ICD-10 (World Health Organization, 1993)) was not so much motivated by its superior validity (as younger colleagues believe), but was founded by a pragmatic consensus. In a polydiagnostic scenario, a patient diagnosed as being 'pre-onset' by one diagnostic system may be already considered as 'postonset' by another system. This notorious 'fuzziness' of the schizophrenia concept makes dating the illness onset not only a psychometric problem, but a theoretical issue intimately associated with the conceptual validity of schizophrenia, that is, what we conceptualise or take schizophrenia to be in the very first place (see Kendler, 1990). Evidence is available suggesting that onset dating is practically impossible in nearly half of those with schizophrenia (Fenton \& McGlashan, 1991).

\section{PSYCHOSIS PREVENTION}

However, since we are here mainly concerned with the feasibility of psychosis prevention, it may seem that the notion of psychosis, at least, is clear-cut, defining a full-blown illness, and therefore pragmatically more useful than the notion of schizophrenia. Unfortunately, the concept of psychosis is theoretically (phenomenologically) not analysed in the DSM and ICD and therefore not adequately defined, apart from a brief list of ostensive indicators (e.g. delusions, hallucinations). There is no conceptualisation of the alterations of human subjectivity (i.e. self-awareness, relatedness to the world, and relatedness to others) that the notion of psychosis appears to convey at a naïve-inituitive level. This is a serious lacuna indeed because the very rationale for early therapeutic intervention rests on the assumption that untreated psychosis exerts toxic effects on the brain (McGlashan \& Johannesen, 1996). Given this conceptual lacuna, however, it appears incomprehensible that certain beliefs should be more dangerous to the brain than other beliefs, that is, a mental state harbouring a false (delusional) rather than a conventional belief should be neurotoxic. This conceptual shortfall becomes of especial clinical significance in the encounter with young patients with insidious onset, marked by the alterations of conduct and personal world-views, but without hallucinations and clear-cut circumscribed delusions.

\section{PRODROMAL PHASE INTERVENTION}

The feasibility of prodromal case identification (in non-clinical and clinical samples) is currently negligible because we largely ignore the pre-onset phenotypes strongly predictive of later schizophrenia. The behavioural 'negative symptoms' are for that purpose prohibitively common (McGorry et al, 1995) and 'without exploring subjective experience, [they] lack the specificity necessary to predict future schizophrenia' (Weiser et al, 2001, p.962). Whereas it is true that negative symptoms were once regarded as the diagnostic indicators of the schizophreniaspectrum disorders, this diagnostic specificity was believed only to emerge upon a more encompassing gestalt, indicative of profound distortions of the patient's subjectivity (Parnas et al, 2002). In the process of operationalisation, they were stripped of their subjective experiential significance and transformed into non-specific behavioural deficits (e.g. a-logia, a-volition, a construal misrepresenting the patient's subjective perspective, often populated by quite 'positive' anomalies of experience: Kring et al, 1993; Danion et al, 2001).

In consequence, only intermittent or low-intensity ('attenuated') psychotic features are the current candidate symptoms for predicting a future full-blown psychosis in first-contact clinical populations (e.g. Miller et al, 2002; McGorry et al, 2002), a predicament that is theoretically highly tautological.

\section{SUBJECTIVE PERSPECTIVE}

The explosion of interest in early intervention has therefore highlighted better than any possible theoretical critique of the contemporary psychopathology, an alarming 
ignorance of the subjective perspectives of pre-schizophrenic and schizophrenic patients. This ignorance is a consequence of many years' domination of the positivisticbehaviourist epistemic dogma (Parnas \& Bovet, 1995) which shuns subjectivity as its core belief. Entire domains of anomalous experience, highly relevant to early differential diagnosis, have vanished from the accepted body of clinical knowledge (e.g. anomalies of self-awareness, identity, temporality, varieties of delusional experience, subtle anomalies in affective, perceptual and cognitive experience), in part also because they cannot be addressed in a simplistic lay vocabulary disconnected from any comprehensive account of human subjectivity. If forced to deal with the subjective dimension none the less, psychiatry has only a commonsensical option at its disposal, in which subjectivity is treated on a par with the spatial objects of the natural world, that is, subjective experience is believed to be describable in the same way that we describe a stone (Parnas $\&$ Zahavi, 2002). Many existing early detection programmes follow a simplified medical model (e.g. advertising for potential patients by publicising lists of 'signal symptoms'), which assumes that the self of the patient, on the one hand, and his or her symptoms, on the other are independent entities (as e.g. in tuberculosis), but which is rather infrequent at the onset of schizophrenia. The onset of schizophrenia is often associated with profound alterations of subjective experience that may modify the patient's world-view in the ontological sense and motivate a quest for metaphysical meaning that cannot be adequately addressed in the medical model terms.

Phenomenologically informed empirical studies, paying attention to anomalies of subjective experience in early schizophrenia and in schizotypal conditions, appear therefore as a promising research direction in the context of early detection. One group of such studies, initiated in Germany, is the 'basic symptoms' research, which targets non-psychotic experiential anomalies of affectivity, cognitionperception and body-motor experience. In a 10-year follow-up of a highly selected clinical sample (suspected of having the potential for schizophrenia), these symptoms predicted subsequent development of schizophrenia with remarkable accuracy (Klosterkötter et al, 2001). These results are obviously in need of replication in more representative clinical samples. Another, overlapping symptom dimension consists of anomalies of self-experience. These

JOSEF PARNAS, MD, Cognitive Research Unit, University Department of Psychiatry, Hvidovre Hospital, DK-2650 Hvidovre, Denmark (e-mail: jpa@cfs.ku.dk) and Danish National Research Foundation, University of Copenhagen, Denmark. E-mail: jpa@cfs.ku.dk

anomalies pre-date the onset of schizophrenia, occur in the schizotypal conditions and differentiate schizophrenia from the psychotic bipolar illness (Parnas et al, 1998, 2003; Møller \& Husby, 2000; Parnas \& Handest, 2003). Guided by phenomenological considerations, it has been recently proposed that alterations of selfawareness constitute the phenotypic core of schizophrenia-spectum disorders (Sass $\&$ Parnas, 2003). Self-anomalies comprise unstable first-person perspective with varieties of depersonalisation, disturbed sense of ownership and agency of experience and action, fluidity of the basic sense of identity, distortions of the stream of consciousness and experiences of disembodiment. Our group at Hvidovre Hospital is finalising an English version of a psychometric instrument targeting these phenomena. However, these symptoms are not suited to rapid, structured screening by non-clinicians, because reliable eliciting and evaluating of these phenotypes require psychopathological sophistication, interviewing skill and considerable training.

\section{CONCLUSION}

The limitations of current clinical knowledge constitute a sufficient reason for not launching large-scale societal programmes for early detection and intervention in schizophrenia. Rather, they should serve as an imperative for further research. However, there is already tremendous room for improvement of ordinary diagnostic practices. Thus, a majority of firstadmission schizophrenia-spectrum patients examined in our studies had one or several pre-admission psychiatric outpatient contacts (e.g. a general practitioner or practising psychiatrist). These patients were usually diagnosed as suffering from affective disorders and offered ineffective treatments. We need to teach mental health professionals how to use our limited clinical resources optimally in order to enhance the accuracy of diagnosis at these crucial early contacts.

\section{REFERENCES}

American Psychiatric Association (1980) Diagnostic and Statistical Manual of Mental Disorders (3rd edn) (DSM-III). Washington, DC: APA.

American Psychiatric Association (1994) Diagnostic and Statistical Manual of Mental Disorders (4th edn) (DSM-IV). Washington, DC: APA.
Danion, J. M., Gokaising, E., Robert, P., et al (200I) Defective relationship between subjective experience and behavior in schizophrenia. American Journal of Psychiatry, I58, 2064-2066.

Fenton, W. S. \& McGlashan, T. H. (1991) Natural

history of schizophrenia subtypes: I. Longitudinal study of paranoid, hebephrenic, and undifferentiated schizophrenia. Archives of General Psychiatry, 48, 969-977. Jansson, L., Handest, P., Nielsen, J., et al (2002) Exploring boundaries of schizophrenia: a comparison of ICD-10 with other diagnostic systems. World Psychiatry, 1/2, 109-114.

Kendler, K. S. (1990) Towards a scientific psychiatric nosology. Archives of General Psychiatry, 47, 969-973.

Klosterkötter, J., Hellmich, M., Steinmyer, E. M., et al (200I) Diagnosing schizophrenia in the initial prodromal phase. Archives of General Psychiatry, 58, 158-164.

Kring, A. M., Kerr, S. L., Smith, D. A., et al (1993) Flat effect in schizophrenia does not reflect diminished subjective experience of emotion. Journal of Abnormal Psychology, 104, 507-517.

McGlashan, T. H. \& Johannesen, J. O. (1996) Early detection and intervention with schizophrenia: rationale. Schizophrenia Bulletin, 22, 20I-222.

McGorry, P. D., McFarlane, C., Patton, G. C., et al (1995) The prevalence of prodromal features of schizophrenia in adolescence: A preliminary study. Acta Psychiatrica Scandinavica, 92, 24I-249.

McGorry, P. D., Yung, A. C., Philips, L. J., et al (2002) Randomized controlled trial of interventions to reduce the risk of progression to first-episode psychosis in clinical sample with subthreshold symptoms. Archives of General Psychiatry, 59, 921-928.

Miller, P., Lawrie, S. M., Byrne, M., et al (2002) Self rated schizotypal cognitions, psychotic symptoms and the onset of schizophrenia in young people at high risk of schizophrenia. Acta Psychiatrica Scandinavica, 105, 34I-346.

Møller, P. \& Husby, R. (2000) The initial prodrome in schizophrenia: searching for naturalistic core dimensions of experience and behavior. Schizophrenia Bulletin, 26, 217-232.

Parnas, J. \& Bovet, P. (1995) Research in psychopathology: epistemologic issues. Comprehensive Psychiatry, 36, 167-|8|.

Parnas, J. \& Zahavi, D. (2002) The role of phenomenology in psychiatric classification and diagnosis. In Psychiatric Diagnosis and Classification (eds M. Maj, W. Gaebel \& J. J. Lopez-lbor), pp. 137-162. World Psychiatric Association Series in Evidence and Experience in Psychiatry. Chichester, UK: John Wiley.

Parnas, J. \& Handest, P. (2003) Phenomenology of anomalous self-experience in early schizophrenia. Comprehensive Psychiatry, 44, 12I-134.

Parnas, J., Jansson, L., Sass, L. A., et al (1998) Selfexperience in the prodromal phases of schizophrenia: A pilot study of first admissions. Neurology, Psychiatry and Brain Research, 6, 107-116.

Parnas, J., Bovet, P. \& Zahavi, D. (2002)

Schizophrenic autism: Clinical phenomenology and pathogenetic implications. World Psychiatry, 1/3, I31-136.

Parnas, J., Handest, P., Sæbye, D., et al (2003)

Anomalies of subjective experience in schizophrenia and bipolarillness. Acta Psychiatrica Scandinavica, 108, 126-133. Sass, L. \& Parnas, J. (2003) Self, consciousness, and schizophrenia. Schizophrenia Bulletin, 29, 427-444. Weiser, M., Reichenberg, A., Rabinowitz, J., et al (200I) Association between nonpsychotic psychiatric diagnoses in adolescent males and subsequent onset of schizophrenia. Archives of General Psychiatry, 58, 959-964. World Health Organization (1993) The ICD-10 Classification of Mental and Behavioural Disorders: Diagnostic Criteria for Research. Geneva: WHO. 